\title{
The flow in periciliary layer in human lungs with Navier-Stokes-Brinkman equations
}

\author{
Nattapol Oangwatcharaparkan and Kanognudge Wuttanachamsri
}

\begin{abstract}
In the human respiratory tract, air breathed in is often contaminated with strange particles such as dust and chemical spray, which may cause people respiratory diseases. However, the human body has an innate immune system that helps to trap the debris by secreting mucus to catch the foreign particles, which are removed from the body by the movement of tiny hairs lining on the surface of the epithelial cells in the immune system. The layer containing the tiny hairs or cilia is called Periciliary Layer (PCL). In this research, we find the velocity of the fluid in the PCL moved by a ciliary beating by using the Navier-Stokes-Brinkman equations. We apply the Galerkin finite element method to determine numerical solutions. For the steady linear case of the equation, the numerical result is in good agreement with an exact solution. Including the time derivative and nonlinear terms, we show that the velocity of the liquid is affected by the velocity of the solid, which follows the physical meaning of the fluid flow. The result can be applied as a bottom boundary condition of the mucous layer to be able to find the velocity of mucus in the human lungs.
\end{abstract}

Keywords. Finite element method, Navier-Stokes-Brinkman equations, cilia, moving solid phases, periciliary layer

\section{Introduction}

In present day air is often contaminated with debris such as dust, smoke and factory pollutions. When we breathe in, they can cause various diseases in our respiratory system acting as an air conveyor into the lungs. Figure 1 illustrates a portion of the respiratory tract and a platform of the mechanical barrier.

Received date: January 11, 2020; Published online: November 10, 2021.

2010 Mathematics Subject Classification. 58F15, 58F17, 53C35.

Corresponding author: Kanognudge Wuttanachamsri. 


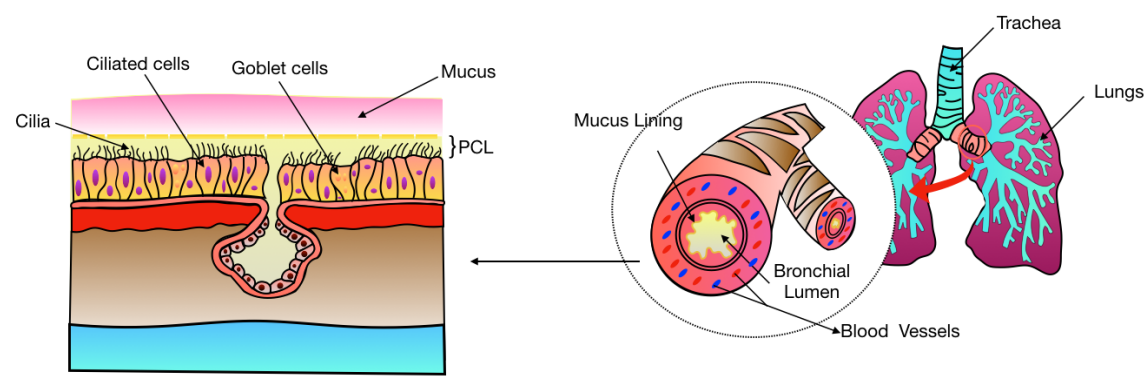

Figure 1: Platform of the mechanical barrier in the respiratory system.

The right of Figure 1 shows trachea, bronchial lumen and lungs and the left of Figure 1 shows cilia, mucus, goblet cells and Periciliary Layer (PCL). While people breathe through the trachea into the lungs, there are also strange particles that get into their bodies. In the meantime, goblet cells in the innate immune system secrete mucus to capture foreign particles. The mucus forms a mucous layer and is removed out of the body by metachronal waving of cilia lining on the ciliated cells. The layer containing cilia is called Periciliary Layer (PCL). The fluid in PCL is named PCL fluid. In this research, we find the velocity of the PCL fluid.

There were several researchers who studied on this problem $[4,7,16,17,20]$. J.R. Cotton and J.W. Grant [7] studied a methodology for performing a mechanical analysis of ciliary bundles by creating a model using a beam theory and a finite element method. P.G. Jayathilake et al. [17] had created a model to determine human pulmonary cilia motion in the PCL. They used the immersed boundary method combined with the projection method and the ciliary forces on the fluid were computed by a direct forcing method. P. Kurbatova et al. [16] studied mucus clearance under normal conditions and in Cystic Fibrosis (CF) patients, who had a genetic disease caused by dehydration of airway surface liquid. They researched on test results from medication that helped to reduce mucus viscosity and created a model of mucociliary clearance. H. Matsui et al. [4] studied the relationship between PCL and mucus by comparing the velocities and showed that the PCL was transported at approximately the same rate as mucus. S.M. Sorin [20] presented a three-dimensional simulation of the formation of metachronal waves in rows of pulmonary cilia. The fluid domains were discretized using a finite volume method and the internal microtubule structure of an individual cilium was modeled using large-deflection and finite-element beams.

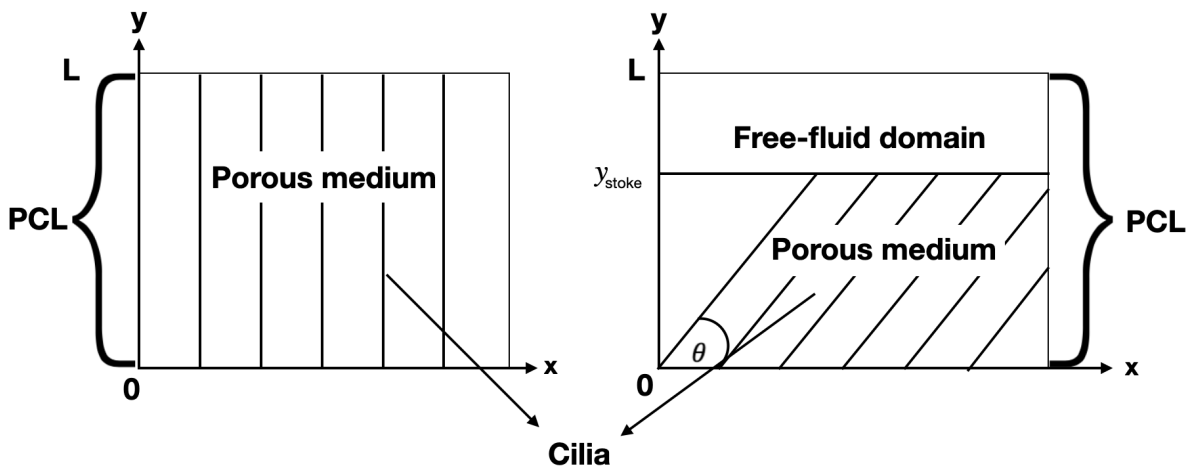

Figure 2: Cartoon picture of cilia making an angle $\theta$ with the horizontal plane. 
In this research, we divide our problem to be two cases. First the cilia make an angle $\theta=90^{\circ}$ with the horizontal plane and second they make an angle $\theta, 40^{\circ} \leq \theta<90^{\circ}$ with the horizontal plane. The left and right of Figure 2 shows the cilia making an angle $\theta=90^{\circ}$ and $\theta<90^{\circ}$ with the horizontal plane, respectively. Because the PCL consists of both PCL fluid and the solid phases, cilia, it is considered as a porous medium. Then when the angle $\theta<90^{\circ}$, the PCL is composed of two domains, the free-fluid region above the porous medium as shown on the right of Figure 2, where $y_{\text {stoke }}$ is the height of the porous domain. We assume that the maximum velocity of cilia occurs when cilia are perpendicular to the horizontal plane and then the velocity decreases and becomes zero when the angle $\theta$ between cilia and the horizontal plane is $40^{\circ}$. Figure 3 shows the fluid flow through the PCL and the profile of the velocity of the fluid in the PCL.

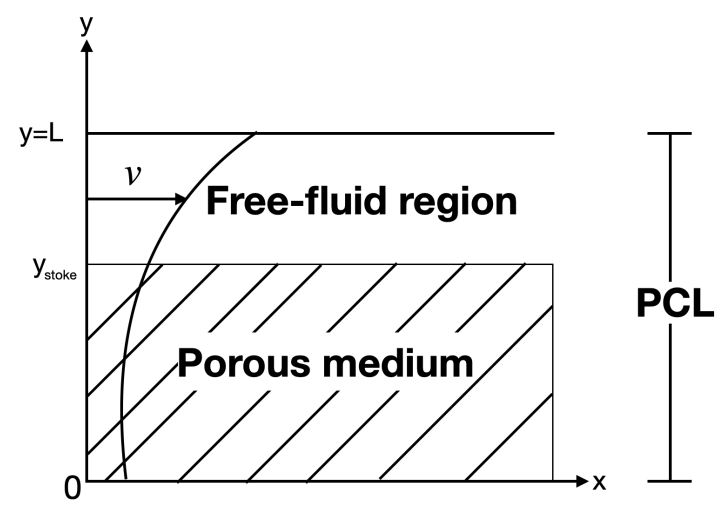

Figure 3: Fluid flow through the PCL and the velocity profile of the PCL fluid.

To study the velocity of the PCL fluid, there were numerous literatures that had used the Navier-Stokes-Brinkman equations or Stokes-Brinkman equations. B. Verleye et al. [1] presented a mathematical model of the permeability of textile by using Navier-Stokes-Brinkman Equations and employed the finite difference method to find the numerical solutions. L. Guta and S. Sundar [13] presented the wave-porous structure interaction by using the Navier-Stokes-Brinkman system and applied the Finite Volume Method (FVM) to calculate the numerical results. O. Iliev et al. [15] presented a numerical subgrid resolution approach for solving the Stokes-Brinkman system of equations for various scientific and industrial problems. B.K. Jha and M.L. Kaurangini [2] presented new approximate analytical solutions of nonlinear Brinkman-Forchheimer externded Darcy model for steady flow in parallel-plates channels filled with porous materials. The system of equations were often solved by using finite element methods [8, 10]. K. Chamsri [10] discretized $n$ dimensional Stokes-Brinkman equations by using a mixed finite element method. K. Chamsri [8] studied the slow flow of a porous medium and adjacent free-fluid region with the Stokes-Brinkman equations. She applied a finite element method to the equations and proved the well-posedness of the discretized equations. X. Xie et al. [22] presented three different methods for solving Darcy-Stokes problem by constructing uniformly stable finite element approximations in two and three dimensions. N. Gatica [3] et al. employed two-dimensional nonlinear Brinkman model with mixed boundary conditions by using a mixed finite element method. P. Kaloni and J. Guo [18] studied the problem of steady nonlinear double-diffusive convection through a porous medium by using Brinkman-Forchheimer model. However, most of the researches mentioned above have not included the solid velocity in their models, where they assume that fluid flows by the pressure gradient. Moreover, although K. Chamsri $[8,10]$ included the solid velocity in her model but 
she had studied only steady linear equations. In this work, we apply one-dimensional NavierStokes-Brinkman equations to our problem in the porous medium domain. We use the classical linearization method for the nonlinear terms and compute the PCL velocity in the two different domains for different angles $\theta$ depending on time $t$, which is more realistic creative problem.

The mathematical model is provided in Section 2. The nonlinear term linearized by using a classical linearization method and model discretization are presented in Section 3. An exact solution is provided in Section 4. Numerical solutions are shown in Section 5, and the conclusion is drawn in Section 6.

\section{Mathematical Model}

In this section, we present our governing equations used in this problem, which are derived by using the Hybrid Mixture Theory (HMT) [14] that helps to transfer a microscale equation to a macroscale equation. Because we consider the cooperative beating of cilia rather than a single cilium, we begin with the momentum equation in the macroscopic scale [9].

$$
\rho \frac{\partial \mathbf{v}^{\mathbf{l}}}{\partial t}+\nabla \cdot\left(\rho \mathbf{v}^{\mathbf{l}} \mathbf{v}^{\mathbf{l}}\right)+\mu \mathbf{k}^{-\mathbf{1}}\left(\epsilon^{l} \mathbf{v}^{\mathbf{l}}-\epsilon^{l} \mathbf{v}^{\mathbf{s}}\right)+\nabla p-\mu\left(\Delta \mathbf{v}^{\mathbf{l}}+\nabla\left(\nabla \cdot \mathbf{v}^{\mathbf{l}}\right)\right)=\rho \mathbf{g},
$$

where $\rho$ is the fluid density; $\epsilon^{l}$ is the porosity which is a constant in this work; $\mathbf{v}^{\mathbf{l}}$ is the velocity of the PCL fluid; $p$ is pressure; $\mathbf{k}^{-\mathbf{1}}$ is the inverse of the permeability tensor; $\mu$ is a dynamic viscosity; $\mathbf{g}$ is the gravity and $\mathbf{v}^{\mathbf{s}}$ is the velocity of the solid phases (cilia) and the continuity equation $[21]$ is

$$
\nabla \cdot\left(\epsilon^{l} \mathbf{v}^{\mathbf{l}}\right)=f
$$

where $f=\frac{\epsilon^{\cdot l}}{\left(1-\epsilon^{l}\right)}+\nabla \cdot \epsilon^{l} \mathbf{v}^{\mathbf{s}}$ and $\epsilon^{\cdot l}$ is the material time derivative of the porosity with respect to the solid phase, $\epsilon^{\cdot l}=\frac{-\left|\mathbf{v}^{\mathbf{s}}\right|}{\xi} \frac{\partial \epsilon^{l}}{\partial \theta}$, where we use the data from [11] to calculate $\frac{\partial \epsilon^{l}}{\partial \theta}$ and $\xi$ is the position along cilia. Multiplying $\epsilon^{l}$ both sides of Eq.(2.1), letting $\mathbf{v}=\epsilon^{l} \mathbf{v}^{\mathbf{l}}$ and substituting Eq.(2.2) into Eq.(2.1), we have

$$
\rho \frac{\partial \mathbf{v}}{\partial t}+\nabla \cdot\left(\frac{\rho \mathbf{v}}{\epsilon^{l}} \mathbf{v}\right)+\epsilon^{l} \mu \mathbf{k}^{-\mathbf{1}} \mathbf{v}-\mu\left(\epsilon^{l}\right)^{2} \mathbf{k}^{-\mathbf{1}} \mathbf{v}^{\mathbf{s}}+\epsilon^{l} \nabla p-\mu \Delta \mathbf{v}-\mu \nabla f=\rho \epsilon^{l} \mathbf{g} .
$$

Since the variables used in this study are the average quantities in the macroscopic scale, for one-dimensional domain we project the governing equation, Eq.(2.3), on the y-axis. Therefore, the pressure gradient is zero in the y-direction. Consequently, the pressure gradient $\frac{d p}{d x}$ is a constant. The velocity and other variables are y-dependent except for the pressure as illustrated in Figure 3. Therefore, for one-dimensional domain, Eq.(2.3) can be rewritten as

$$
\rho \frac{\partial v}{\partial t}+\frac{2 \rho}{\epsilon^{l}} v \frac{\partial v}{\partial y}+\epsilon^{l} \mu k^{-1} v-\mu\left(\epsilon^{l}\right)^{2} k^{-1} v^{s}+\epsilon^{l} \frac{d p}{d x}-\mu \frac{\partial^{2} v}{\partial y^{2}}-\mu \frac{\partial f}{\partial y}=\rho \epsilon^{l} g,
$$

where $v$ and $k$ are the velocity and permeability in the x-direction, respectively. The boundary conditions used in this work are

$$
\begin{aligned}
v(0) & =0 \\
v^{\prime}(L) & =0,
\end{aligned}
$$

where we suppose that the base of cilia does not move and the maximum velocity is occurred at the tips of cilia, where $0<y<L$, and $L$ is the length of our numerical domain. The velocity of 
the solid phases can be extracted from the experimental data in [19], which is presented in [12] as polynomials of degree 8 depending on the position along cilia $\xi$,

$$
v^{s}=c_{8} \xi^{8}+c_{7} \xi^{7}+c_{6} \xi^{6}+c_{5} \xi^{5}+c_{4} \xi^{4}+c_{3} \xi^{3}+c_{2} \xi^{2}+c_{1} \xi
$$

where $\xi=\frac{y}{\sin \theta}$; the variable $\theta$ is the angle between the cilia and the horizontal plane; the coefficients $c_{i}, i=1,2, \ldots, 8$ are constants. Then Eq.(2.4) becomes

$$
\begin{aligned}
& \rho \frac{\partial v}{\partial t}+\frac{2 \rho}{\epsilon^{l}} v \frac{\partial v}{\partial y}+\epsilon^{l} \mu k^{-1} v-\mu \frac{\partial^{2} v}{\partial y^{2}}=\mu\left(\epsilon^{l}\right)^{2} k^{-1} c_{8}\left(\frac{y}{\sin \theta}\right)^{8}+\mu\left(\epsilon^{l}\right)^{2} k^{-1} c_{7}\left(\frac{y}{\sin \theta}\right)^{7} \\
& +\ldots+\mu\left(\epsilon^{l}\right)^{2} k^{-1} c_{2}\left(\frac{y}{\sin \theta}\right)^{2}+\mu\left(\epsilon^{l}\right)^{2} k^{-1} c_{1}\left(\frac{y}{\sin \theta}\right)-\epsilon^{l} \frac{d p}{d x}+\mu \frac{\partial f}{\partial y}+\rho \epsilon^{l} g, \quad 0<y<L .
\end{aligned}
$$

Now we have a one-dimensional governing equation, which is discretized in the next section and the numerical results in the porous medium are presented in Section 5 .

\section{Model Discretization}

In this section, we discretize Eq.(2.7) by using the Galerkin finite element method. Before discretizing the model, we first linearize the nonlinear term, $\frac{2 \rho}{\epsilon^{l}} v \frac{\partial v}{\partial y}$, with the classical linearization method [5]. That is

$$
v \frac{\partial v}{\partial y} \approx\left(v^{*}\right) \frac{\partial v}{\partial y}
$$

where $v^{*}$ is an approximation of $v$. In this work we assume that

$$
v^{*}=y \text {. }
$$

Therefore Eq.(2.7) can be rewritten as

$$
\begin{aligned}
& \rho \frac{\partial v}{\partial t}+\frac{2 \rho}{\epsilon^{l}} y \frac{\partial v}{\partial y}+\epsilon^{l} \mu k^{-1} v-\mu \frac{\partial^{2} v}{\partial y^{2}}=\mu\left(\epsilon^{l}\right)^{2} k^{-1} c_{8}\left(\frac{y}{\sin \theta}\right)^{8}+\mu\left(\epsilon^{l}\right)^{2} k^{-1} c_{7}\left(\frac{y}{\sin \theta}\right)^{7} \\
& +\ldots+\mu\left(\epsilon^{l}\right)^{2} k^{-1} c_{2}\left(\frac{y}{\sin \theta}\right)^{2}+\mu\left(\epsilon^{l}\right)^{2} k^{-1} c_{1}\left(\frac{y}{\sin \theta}\right)-\epsilon^{l} \frac{d p}{d x}+\mu \frac{\partial f}{\partial y}+\rho \epsilon^{l} g .
\end{aligned}
$$

The weak formulation of the model after the domain is discretized to be $n$ elements is

$$
\begin{aligned}
& \sum_{i=1}^{n} \int_{y_{i}}^{y_{i+1}}\left(w \rho \frac{\partial v}{\partial t}\right) d y+\sum_{i=1}^{n} \int_{y_{i}}^{y_{i+1}}\left(\frac{2 w \rho}{\epsilon^{l}} y \frac{\partial v}{\partial y}+w \epsilon^{l} \mu k^{-1} v+\mu \frac{\partial w}{\partial y} \frac{\partial v}{\partial y}\right) d y \\
& =\sum_{i=1}^{n} \int_{y_{i}}^{y_{i+1}} w\left(\mu\left(\epsilon^{l}\right)^{2} k^{-1} c_{8}\left(\frac{y}{\sin \theta}\right)^{8}+\mu\left(\epsilon^{l}\right)^{2} k^{-1} c_{7}\left(\frac{y}{\sin \theta}\right)^{7}+\ldots\right. \\
& \left.+\mu\left(\epsilon^{l}\right)^{2} k^{-1} c_{2}\left(\frac{y}{\sin \theta}\right)^{2}+\mu\left(\epsilon^{l}\right)^{2} k^{-1} c_{1}\left(\frac{y}{\sin \theta}\right)-\epsilon^{l} \frac{d p}{d x}+\rho \epsilon^{l} g\right) d y \\
& \left.-\mu \sum_{i=1}^{n} \int_{y_{i}}^{y_{i+1}} f \frac{\partial w}{\partial y} d y+\mu[w f]_{0}^{L}+\mu\left[w \frac{\partial v}{\partial y}\right]_{0}^{L}\right),
\end{aligned}
$$

where $w \in H_{0}^{1}(\Omega)$ is a weight function, $\Omega=[0, L]$ is our domain and $y_{i}=1,2, \ldots, n$, are nodes in $\Omega$. We discretize the velocity $v$ by using the linear shape functions,

$$
\begin{aligned}
& H_{1}=\frac{y_{i+1}-y}{h_{i}}, \\
& H_{2}=\frac{y-y_{i}}{h_{i}} .
\end{aligned}
$$


Then the velocity in each element is

$$
v(y, t)=H_{1}(y) v_{i}(t)+H_{2}(y) v_{i+1}(t),
$$

where $v_{i}(t)=v\left(y_{i}, t\right)$ and $v_{i+1}(t)=v\left(y_{i+1}, t\right)$ and

$$
h_{i}=y_{i+1}-y_{i}
$$

The Equation (3.3), without the summation, can be rewritten as

$$
\left[M_{e}\right]\left[\dot{v}_{e}\right]+\left[K_{e}\right][v]=\left[f_{e}\right],
$$

where $\dot{v}_{e}$ is the derivative of the velocity in an element domain with respect to time,

$$
\begin{gathered}
{\left[M_{e}\right]\left[\dot{v}_{e}\right]=\int_{y_{i}}^{y_{i+1}}\left(\left[\begin{array}{l}
H_{1} \\
H_{2}
\end{array}\right] \rho \frac{\partial v}{\partial t}\right) d y=\rho \frac{h_{i}}{6}\left[\begin{array}{ll}
2 & 1 \\
1 & 2
\end{array}\right]\left[\begin{array}{c}
\dot{v}_{i} \\
\dot{v}_{i+1}
\end{array}\right],} \\
{\left[K_{e}\right]=\left(\frac{\rho}{3 \epsilon^{l}} \frac{1}{h_{i}^{2}}\left[\begin{array}{ll}
-\left(y_{i+1}^{3}-3 y_{i+1} y_{i}^{2}+2 y_{i}^{3}\right) & \left(y_{i+1}^{3}-3 y_{i+1} y_{i}^{2}+2 y_{i}^{3}\right) \\
-\left(2 y_{i+1}^{3}-3 y_{i+1}^{2} y_{i}+y_{i}^{3}\right) & \left(2 y_{i+1}^{3}-3 y_{i+1}^{2} y_{i}+y_{i}^{3}\right)
\end{array}\right],\right.} \\
\left.+\frac{\epsilon^{l} \mu k^{-1}}{6} h_{i}\left[\begin{array}{ll}
2 & 1 \\
1 & 2
\end{array}\right]+\frac{\mu}{h_{i}}\left[\begin{array}{cc}
1 & -1 \\
-1 & 1
\end{array}\right]\right)\left[\begin{array}{c}
v_{i} \\
v_{i+1}
\end{array}\right]
\end{gathered}
$$

and

$$
\begin{aligned}
{\left[f_{e}\right] } & =\int_{y_{i}}^{y_{i+1}}\left[\begin{array}{c}
H_{1} \\
H_{2}
\end{array}\right]\left(\mu\left(\epsilon^{l}\right)^{2} k^{-1} c_{8}\left(\frac{y}{\sin \theta}\right)^{8}+\mu\left(\epsilon^{l}\right)^{2} k^{-1} c_{7}\left(\frac{y}{\sin \theta}\right)^{7}+\ldots\right. \\
& \left.+\mu\left(\epsilon^{l}\right)^{2} k^{-1} c_{2}\left(\frac{y}{\sin \theta}\right)^{2}+\mu\left(\epsilon^{l}\right)^{2} k^{-1} c_{1}\left(\frac{y}{\sin \theta}\right)-\epsilon^{l} \frac{d p}{d x}+\rho \epsilon^{l} g\right) d y \\
& -\mu \sum_{i=1}^{n} \int_{y_{i}}^{y_{i+1}}\left[\begin{array}{c}
H_{1}^{\prime} \\
H_{2}^{\prime}
\end{array}\right] f d y+\mu\left[f\left[\begin{array}{l}
H_{1} \\
H_{2}
\end{array}\right]\right]_{0}^{L}+\mu\left[\frac{\partial v}{\partial y}\left[\begin{array}{c}
H_{1} \\
H_{2}
\end{array}\right]\right]_{0}^{L} .
\end{aligned}
$$

Assembling the element matrices, we have

$$
[M][\dot{v}]+[K][v]=[F],
$$

where $[M],[K]$ and $[F]$ are the assembly matrices of $\left[M_{e}\right],\left[K_{e}\right]$ and $\left[F_{e}\right]$, respectively.

For the time derivative term in Eq.(3.11) we use backward difference method. Then Eq.(3.11) can be written as

$$
[M][\dot{v}]^{t+\Delta t}+[K][v]^{t+\triangle t}=[F]^{t+\triangle t}
$$

where $\Delta t$ is a time difference and

$$
[\dot{v}]^{t+\triangle t}=\frac{v^{t+\triangle t}-v^{t}}{\triangle t} .
$$

Substituting Eq.(3.13) into Eq.(3.12), we have

$$
\begin{gathered}
{[M]\left[\frac{v^{t+\Delta t}-v^{t}}{\triangle t}\right]+[K][v]^{t+\Delta t}=[F]^{t+\Delta t},} \\
{[M]\left[v^{t+\Delta t}-v^{t}\right]+\triangle t[K][v]^{t+\Delta t}=\triangle t[F]^{t+\triangle t},} \\
([M]+\triangle t[K])[v]^{t+\triangle t}=\Delta t[F]^{t+\triangle t}+[M][v]^{t} .
\end{gathered}
$$

Next, we solve the discretized equation and compare our results with an exact solution provided in the next section. 


\section{Exact Solution}

To verify our numerical solution, in this section, we provide an exact solution by considering only the steady and linear terms of the governing equation without the velocity of the solid phases,

$$
\frac{d p}{d x}=-\mu k^{-1} v+\frac{\mu}{\epsilon^{l}} \frac{d^{2} v}{d y^{2}}
$$

with the boundary conditions

$$
\begin{aligned}
v(1) & =1 \\
\left.\frac{d v}{d y}\right|_{y=y_{\text {stoke }}^{-}} & =\left.\frac{d v}{d y}\right|_{y=y_{\text {stoke }}^{+}},
\end{aligned}
$$

where we use $L=1$. The exact solution of Eqs.(4.1)-(4.3) is obtained from [6], which is

$$
v(y)=\frac{1}{1+\left(\frac{1-y_{\text {stoke }}}{\sqrt{k}}\right)} \begin{cases}1+\left(\frac{y-y_{\text {stoke }}}{\sqrt{k}}\right) & , y>y_{\text {stoke }} \\ e^{\frac{y-y_{\text {stoke }}}{\sqrt{k}}} & , y<y_{\text {stoke }}\end{cases}
$$

We then compare the exact solution with our numerical solutions in the next section.

\section{$5 \quad$ Numerical Results}

In this section, we provide our numerical results of the governing equation, Eq.(2.7). Since our model depends on the angle $\theta$, in this study, we find the solutions when $\theta=50^{\circ}, 60^{\circ}, 70^{\circ}, 80^{\circ}$ and $90^{\circ}$ and assume that the forward stroke of cilia stops at $\theta=40^{\circ}$ [19]. The coefficients of the polynomials of degree 8 , the velocities of cilia, for each angle $\theta$ are provided in Table 1 [12]. The solid velocities are drawn in Figure 4 where $\xi$ used in this study is a dimensionless quantity, which is the distance along cilia divided by the cilia length. That is $0 \leq \xi \leq 1$ with the unit of $\xi$ is equal to 1 . Notice that the maximum velocity of cilia is assumed to occur at $\theta=90^{\circ}$ at the tips of cilia. The other values in the Navier-Stokes-Brinkman equations are $g=9.8 \times 10^{6}\left(\mu \mathrm{m} / \mathrm{s}^{2}\right)$, $\rho=9.922 \times 10^{-13}\left(\mathrm{~g} / \mu \mathrm{m}^{3}\right), \mu=3 \times 10^{-6}(\mathrm{~g} /(\mu \mathrm{m})(\mathrm{s})), \frac{d p}{d x}=-1 \times 10^{-9}\left(\mathrm{~g} /\left(\mu \mathrm{m}^{2}\right)\left(\mathrm{s}^{2}\right)\right), \epsilon^{l}=$ $0.5223 \theta^{5}-3.0283 \theta^{4}+7.0630 \theta^{3}-8.4987 \theta^{2}+5.5056 \theta-0.8627$ and $k^{-1}$ is shown in Table 2 , where the values of $\epsilon^{l}$ and $k^{-1}$ are obtained from [12] and [11], respectively. The unit of $\epsilon^{l}$ is equal to $1,\left[\epsilon^{l}\right]=1$, and the unit of $k^{-1}$ is $\frac{1}{\mu m^{2}}$. 
Table 1: The eighth-order polynomial functions: $v^{s}=c_{8} \xi^{8}+c_{7} \xi^{7}+c_{6} \xi^{6}+c_{5} \xi^{5}+c_{4} \xi^{4}+$ $c_{3} \xi^{3}+c_{2} \xi^{2}+c_{1} \xi$ for the angels $\theta=50^{\circ}, 60^{\circ}, 70^{\circ}, 80^{\circ}$ and $90^{\circ}$.

\begin{tabular}{|c|c|c|c|c|c|}
\hline Coefficient & \multicolumn{5}{|c|}{ degree } \\
\hline $10^{5} \times$ & $50^{\circ}$ & $60^{\circ}$ & $70^{\circ}$ & $80^{\circ}$ & $90^{\circ}$ \\
\hline$c_{8}$ & 0.2498 & 0.4043 & -0.4987 & -0.3648 & -0.5386 \\
\hline$c_{7}$ & -1.0781 & -1.6788 & 2.1268 & 1.5687 & 2.2148 \\
\hline$c_{6}$ & 1.9290 & 2.8656 & -3.7102 & -2.7659 & -3.7309 \\
\hline$c_{5}$ & -1.8459 & -2.5945 & 3.4021 & 2.5751 & 3.3198 \\
\hline$c_{4}$ & 1.0133 & 1.3380 & -1.7529 & -1.3584 & -1.6788 \\
\hline$c_{3}$ & -0.3157 & -0.3896 & 0.5012 & 0.4022 & 0.4803 \\
\hline$c_{2}$ & 0.0504 & 0.0585 & -0.0717 & -0.0593 & -0.0694 \\
\hline$c_{1}$ & -0.0023 & -0.0024 & 0.0049 & 0.0044 & 0.0050 \\
\hline
\end{tabular}

Table 2: The inverse of the permeability tensor, $k^{-1}$, for the angles $\theta=50^{\circ}, 60^{\circ}, 70^{\circ}, 80^{\circ}$ and $90^{\circ}$.

\begin{tabular}{|c|c|}
\hline angle $\theta$ & $\begin{array}{c}\text { The inverse of the permeability tensor, } k^{-1}, \\
{\left[k^{-1}\right]=1 /(\mu \mathrm{m})^{2}}\end{array}$ \\
\hline $50^{\circ}$ & $1 / 0.0019$ \\
\hline $60^{\circ}$ & $1 / 0.0020$ \\
\hline $70^{\circ}$ & $1 / 0.0022$ \\
\hline $80^{\circ}$ & $1 / 0.0023$ \\
\hline $90^{\circ}$ & $1 / 0.0024$ \\
\hline
\end{tabular}

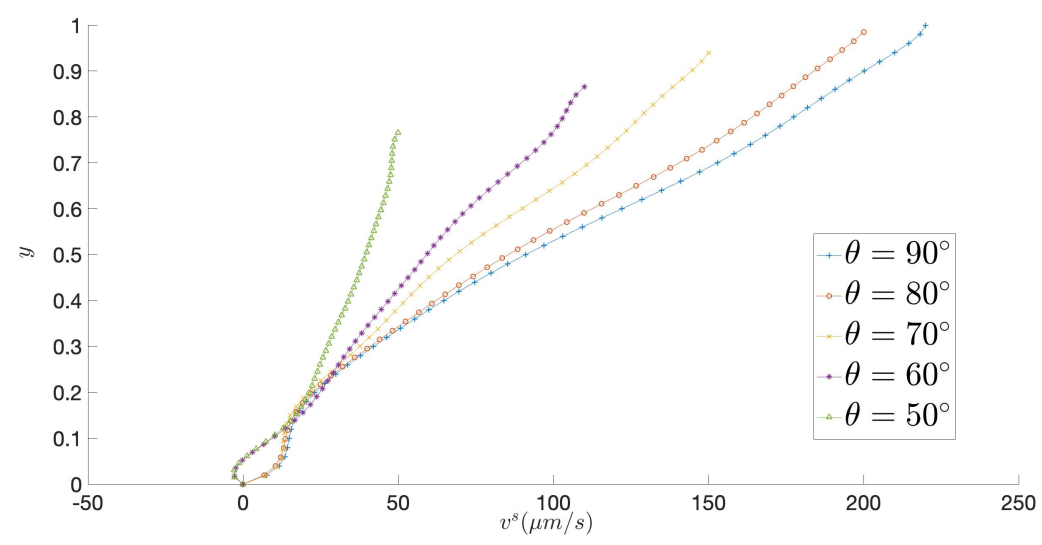

Figure 4: The velocities of cilia when the angles $\theta=50^{\circ}, 60^{\circ}, 70^{\circ}, 80^{\circ}$ and $90^{\circ}$. (For interpretation of the references to color in this figure legend, the reader is referred to the free web version of this article.)

We first verify our numerical solution by comparing the result with the exact solution as 
shown in Figure 5 . The velocities are calculated at the angle $\theta=90^{\circ}$ with the boundary conditions Eq.(4.2). The numerical results are provided with 10,20,40 and 100 number of elements. Notice that the numerical solutions converge to the exact solution when the number of elements increases. The $l_{2}$-norm errors of the numerical solutions for each number of elements are illustrated in Table 3. It shows that the more number of elements we have, the less error we obtain.

Table 3: The $l_{2}$-norm errors of the numerical solutions.

\begin{tabular}{|c|c|c|c|c|}
\hline number of elements & 10 elements & 20 elements & 40 elements & 100 elements \\
\hline$l_{2}$-norm errors & 0.0860 & 0.0302 & 0.0102 & 0.0025 \\
\hline
\end{tabular}

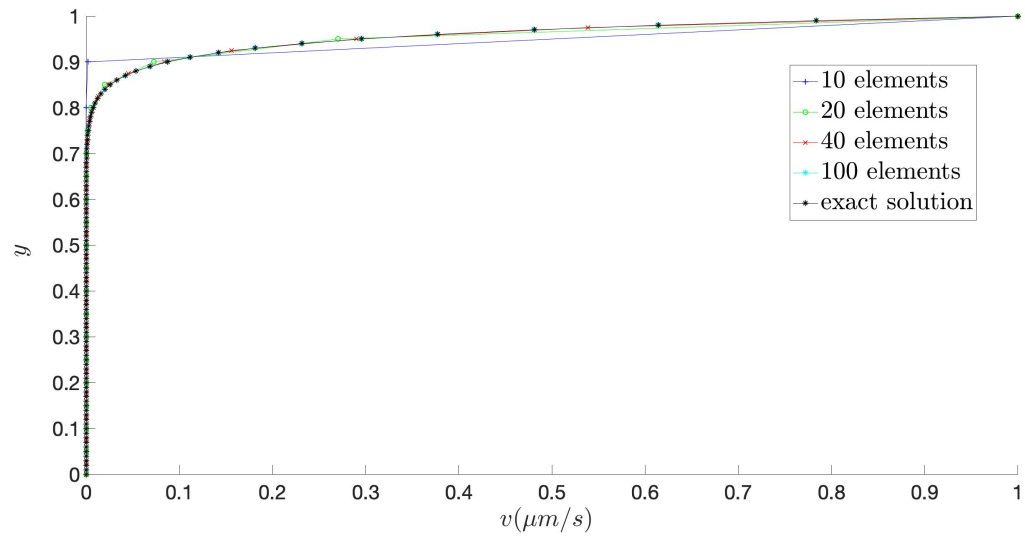

Figure 5: The exact solution and our numerical solutions with 10, 20, 40 and 100 number of elements. (For interpretation of the references to color in this figure legend, the reader is referred to the free web version of this article.)

Next, we calculate the velocities of the PCL fluid for the angles $\theta=50^{\circ}, 60^{\circ}, 70^{\circ}, 80^{\circ}$ and $90^{\circ}$ with the boundary conditions $v(0)=0$ and $v^{\prime}(L)=0$. In this work, we use dimensionless length. So we let $L=1$. Since the PCL velocity obtained from Eq.(3.16) is $v=\epsilon^{l} v^{l}$, which is called Darcy velocity, we present both $v^{l}$ and $v$ in Figures 6 and 7 , respectively. From left to right and from top to bottom, Figures 6 and 7 show the velocities of the PCL fluid from the angles $50^{\circ}$ to $90^{\circ}$ respectively. Since our governing equation depends on time, we let $\triangle t=0.1$ and assume that the cilia perpendicular to the horizontal plane when $t=0$. Then the cilia bend down from angle $\theta=90^{\circ}$ to the angle $\theta=50^{\circ}$ and stop beating at $\theta=40^{\circ}$. We assume that the time difference for each angle is $\triangle t=0.1$. Therefore, when $t=0.4$ the cilia make angle $\theta=50^{\circ}$ with the horizontal plane. In Figure 6 , we compare the velocity of PCL fluid with the velocity of cilia. Notice that the velocity, $v^{l}$ and the velocity of cilia of each angle $\theta$ are almost identical. The $l_{2}$-norm errors of the graphs are shown in Table 4 , where the values are between 0.2 and 0.5. Figure 7 shows the Darcy velocities of the PCL fluid and the velocities of the cilia when the angles $\theta=50^{\circ}, 60^{\circ}, 70^{\circ}, 80^{\circ}$ and $90^{\circ}$. The Darcy velocity is less than the velocity of cilia for each angle $\theta$, which follows the physical meaning the velocity of the solid phase should be greater than the velocity of the fluid phase. The Darcy velocities of the PCL fluid of all angles are plotted together in Figure 8. The maximum velocity occurs at $\theta=90^{\circ}$ and the velocity decreases when the angle decreases. To be useful in practice, the velocities are approximated 
by using the polynomials of degree 12 . From left to right, the graphs illustrate the velocities of the angles $\theta=50^{\circ}, 60^{\circ}, 70^{\circ}, 80^{\circ}$ and $90^{\circ}$, respectively. The coefficients of each polynomials are provided in Table 5 , where $\xi$ is the position along cilia. The $l_{2}$-norm errors of the Darcy velocities of the PCL fluid and their polynomial approximations of degree 12 are shown in Table 6 , which are about 0.001 . It shows that the velocities and the polynomial approximations are almost indistinguishable. Notice that the maximum Darcy velocity of the PCL fluid is about $82 \%$ of the maximum velocity of cilia.

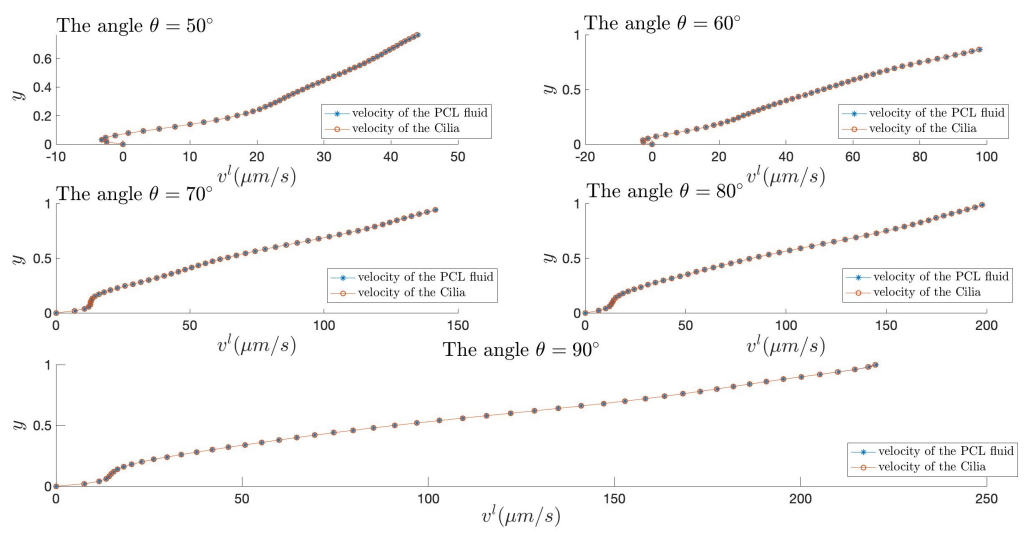

Figure 6: The velocities of the PCL fluid and cilia when the angles $\theta=50^{\circ}, 60^{\circ}, 70^{\circ}, 80^{\circ}$ and $90^{\circ}$. (For interpretation of the references to color in this figure legend, the reader is referred to the free web version of this article.)

Table 4: The $l_{2}$-norm errors of the velocities of the PCL fluid and the velocities of cilia.

\begin{tabular}{|c|c|}
\hline angle $\theta$ & $l_{2}$-norm error \\
\hline $50^{\circ}$ & 0.2503 \\
\hline $60^{\circ}$ & 0.2872 \\
\hline $70^{\circ}$ & 0.2984 \\
\hline $80^{\circ}$ & 0.3414 \\
\hline $90^{\circ}$ & 0.4770 \\
\hline
\end{tabular}




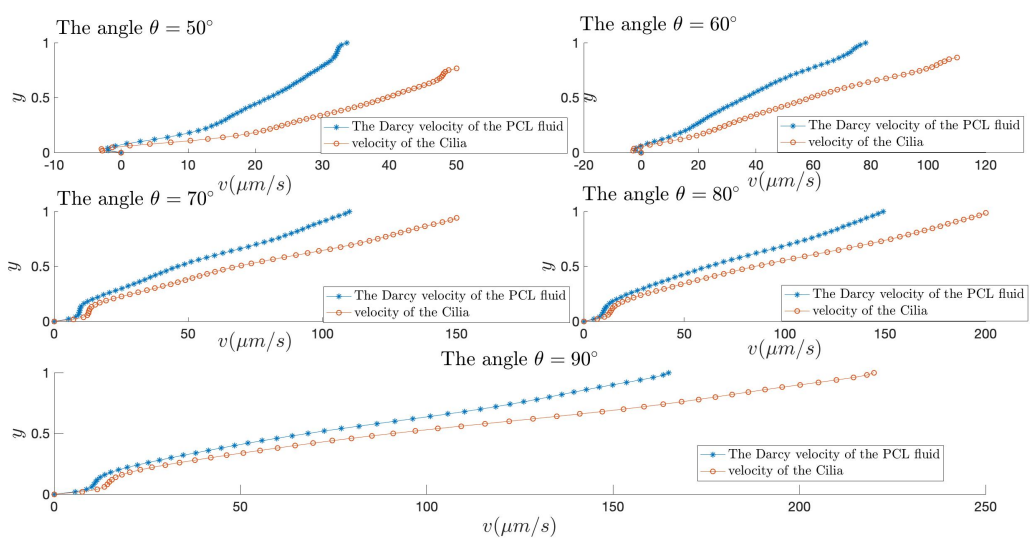

Figure 7: The Darcy velocities of the PCL fluid and the velocities of cilia when the angles $\theta=50^{\circ}, 60^{\circ}, 70^{\circ}, 80^{\circ}$ and $90^{\circ}$. (For interpretation of the references to color in this figure legend, the reader is referred to the free web version of this article.)

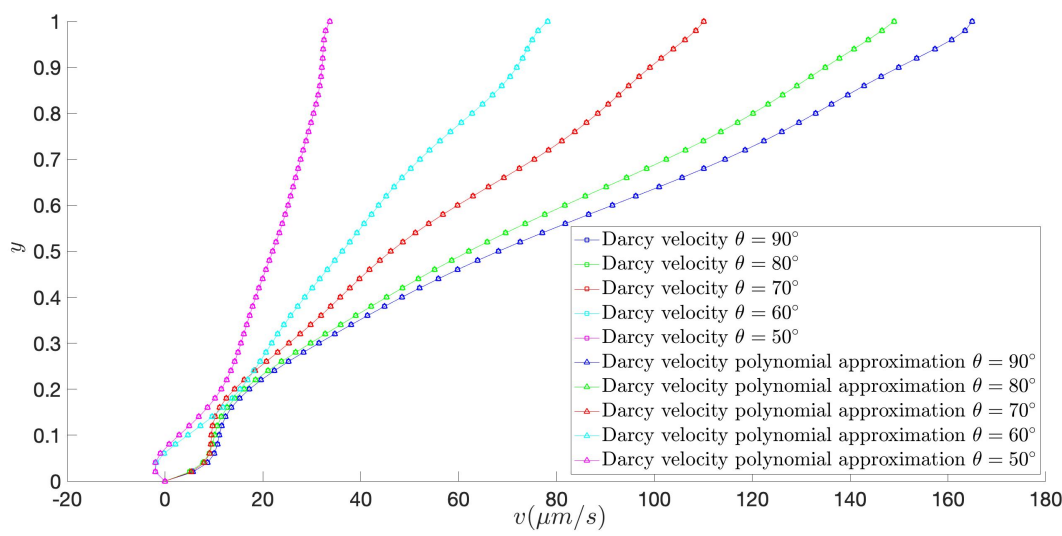

Figure 8: The Darcy velocities of the PCL fluid and the polynomial approximations of the Darcy velocities when $\theta=50^{\circ}, 60^{\circ}, 70^{\circ}, 80^{\circ}$ and $90^{\circ}$. (For interpretation of the references to color in this figure legend, the reader is referred to the free web version of this article.) 
Table 5: The twelfth-order polynomial functions: $v=c_{12} \xi^{12}+c_{11} \xi^{11}+c_{10} \xi^{10}+c_{9} \xi^{9}+$ $c_{8} \xi^{8}+c_{7} \xi^{7}+c_{6} \xi^{6}+c_{5} \xi^{5}+c_{4} \xi^{4}+c_{3} \xi^{3}+c_{2} \xi^{2}+c_{1} \xi$ for the angels $\theta=50^{\circ}, 60^{\circ}, 70^{\circ}, 80^{\circ}$ and $90^{\circ}$.

\begin{tabular}{|c|c|c|c|c|c|}
\hline Coefficient & \multicolumn{5}{|c|}{ degree } \\
\hline $10^{5} \times$ & $50^{\circ}$ & $60^{\circ}$ & $70^{\circ}$ & $80^{\circ}$ & $90^{\circ}$ \\
\hline$c_{12}$ & 0.0108 & 0.0155 & -0.0118 & -0.0081 & -0.0116 \\
\hline$c_{11}$ & -0.0669 & -0.0950 & 0.0771 & 0.0539 & 0.0738 \\
\hline$c_{10}$ & 0.1868 & 0.2621 & -0.2266 & -0.1615 & -0.2126 \\
\hline$c_{9}$ & -0.3109 & -0.4314 & 0.3961 & 0.2870 & 0.3653 \\
\hline$c_{8}$ & 0.5114 & 0.7588 & -0.8242 & -0.6089 & -0.8210 \\
\hline$c_{7}$ & -0.9908 & -1.5541 & 1.9310 & 1.4447 & 1.9945 \\
\hline$c_{6}$ & 1.4452 & 2.2355 & -2.9371 & -2.2221 & -2.9894 \\
\hline$c_{5}$ & -1.3010 & -1.9231 & 2.5858 & 1.9862 & 2.5686 \\
\hline$c_{4}$ & 0.6984 & 0.9732 & -1.3123 & -1.0318 & -1.2817 \\
\hline$c_{3}$ & -0.2155 & -0.2810 & 0.3727 & 0.3034 & 0.3645 \\
\hline$c_{2}$ & 0.0343 & 0.0420 & -0.0531 & -0.0446 & -0.0525 \\
\hline$c_{1}$ & -0.0016 & -0.0017 & 0.0036 & 0.0033 & 0.0038 \\
\hline
\end{tabular}

Table 6: The $l_{2}$-norm errors of the Darcy velocities of the PCL fluid and the twelfth-order polynomial approximations of each angle $\theta$.

\begin{tabular}{|c|c|}
\hline angle $\theta$ & $l_{2}$-norm error $\left(\times 10^{-3}\right)$ \\
\hline $50^{\circ}$ & 0.1205 \\
\hline $60^{\circ}$ & 0.1426 \\
\hline $70^{\circ}$ & 0.1738 \\
\hline $80^{\circ}$ & 0.1020 \\
\hline $90^{\circ}$ & 0.0824 \\
\hline
\end{tabular}

\section{Conclusion}

We consider the fluid flow in the periciliary layer (PCL) in the human respiratory system. The PCL consists of two domains: the porous medium and the free-fluid domain above the porous layer. However, when cilia make an angle $\theta=90^{\circ}$ with the horizontal plane, the PCL domain contains only the porous medium. In the porous medium domain we use unsteady nonlinear Brinkman equation and in the free-fluid domain we use Navier-Stokes equation. Without the time derivative and nonlinear terms, we compare our numerical results with the exact solution with a good agreement. The numerical solutions converge to the exact solution when the number of elements increases, see Table 3. With the nonlinear term, it is solved by the classical linearization method and then we use the Galerkin finite element method to find the numerical solutions. For the time derivative term, we use the Backward Difference method. The numerical solutions of the Navier-Stokes-Brinkman equations are computed for different angles $\theta=50^{\circ}, 60^{\circ}, 70^{\circ}, 80^{\circ}$ and $90^{\circ}$ when $\triangle t=0.1$. The Darcy velocity $v$ and the velocity $v^{l}$ are compared with the velocity of cilia. As expected, they follow the physical meaning. That is the velocity $v^{l}$ is almost iden- 
tical to the velocity of cilia, see Table 4, and the Darcy velocity is about $23 \%$ slower than the velocity of cilia (see Figure 7). For useful convenience in practice, the Darcy velocities are approximated by using the polynomials of degree 12 , where the coefficients are provided in Table 5 . The $l_{2}$-norm errors of the approximation are shown in Table 6 , which are about 0.001 for each angle $\theta$.

\section{Acknowledgments}

This work is supported by the School of Science, King Mongkut's Institute of Technology Ladkrabang, Thailand.

\section{References}

[1] B. Verleye, M. Klitz, R. Croce, M. Griebel, S. V. Lomov, D. Roose, and I. Verpoest, Predicting the Termeability of Textile Reinforcements Via a Hybrid Navier-Stokes/Brinkman Solver, Proceedings of the 8th international conference on flow processes in composite materials, (2006), 65-72.

[2] B. K. Jha and M. L. Kaurangini, Approximate Analytical Solutions for the Nonlinear Brinkman-Forchheimer- Extended Darcy Flow Model, Applied Mathematics, 2 (2011), 14321436 .

[3] G.N. Gatica, L.F. Gatica and F.A. Sequeira, Analysis of an Augmented Pseudostress-Based Mixed Formulation for a Nonlinear Brinkman Model of Porous Media Flow, Computer Methods in Applied Mechanics and Engineering, 289 (2015), 104-130.

[4] H. Matsui, S. H. Randell, S. W. Peretti, C. W. Davis and R. C. Boucher, Coordinated Clearance of Periciliary Liquid and Mucus from Airway Surfaces, The Journal of clinical investigation, 102 (1998), 1125-1131.

[5] H. B. Young and W. Kwan, The Finite Element Method Using MATLAB, CRC Press, (1997).

[6] J. Koplik, H. Levine and A. Zee, Viscosity Renormalization in the Brinkman Equation, The Physics of fluids, 26 (1983), 2864-2870.

[7] J. R. Cotton and J. W. Grant, A Finite Element Method for Mechanical Response of Hair Cell Ciliary Bundlez, Journal of biomechanical engineering, 122 (2000), 44-50.

[8] K. Chamsri, Formulation of a Well-Posed Stokes-Brinkman Problem with a Permeability Tensor, Journal of Mathematics, 1 (2015), 1-7.

[9] K. Chamsri, Modeling the Flow of PCL Fluid due to the Movement of Lung Cilia, Ph.D thesis, University of Colorado Denver, 2012.

[10] K. Chamsri, N-Dimensional Stokes-Brinkman Equations using a Mixed Finite Element Method, Australian Journal of Basic and Applied Sciences, 8(11) Special (2014), 30-36.

[11] K. Chamsri and L.S. Benethum, Permeability of Fluid Flow Through a Preiodic Array of Cylinders, Applied Mathematical Modelling, 39(1) (2015), 244-254. 
[12] K. Wuttanachamsri and L. Schreyer, Effects of Cilia Movement on Fluid Velocity: II Numerical Solutions over a Fixed Domain, Transport in Porous Media, 134 (2020), 471489 .

[13] L. Guta and S. Sundar, Navier-Stokes-Brinkman System for Interaction of Viscous Waves with a Submerged Porous Structure, Tamkang journal of mathematics, 41 (2010), 217-243.

[14] L.S. Benethum and J.H. Cushman, Multiphase, Hybrid Mixture Theory for Swelling Systems-I: Balance Laws, International Journal of Engineering Science, 34 (1996), 125145 .

[15] O. Iliev, Z. Lakdawala and V. Starikovicius, On a Numerical Subgrid Upscaling Algorithm for Stokes-Brinkman Equations, Computers \& Mathematics with Applications, 65 (2013), $435-448$.

[16] P. Kurbatova, N. Bessonov, V. Volpert, H. Tiddens, C. Cornu, P. Nony and D. Caudri, Model of Mucociliary Clearance in Cystic Fibrosis Lungs, Journal of theoretical biology, 372 (2015), 81-88.

[17] P. G. Jayathilake, Z. Tan, D. V. Le, H. P. Lee and B.C. Khoo, Three-Dimensional Numerical Simulations of Human Pulmonary Cilia in The Periciliary Liquid Layer by the Immersed Boundary Method, Computers \& Fluids, 67 (2012), 130-137.

[18] P. N. Kaloni and J. Guo, Steady Nonlinear Double-Diffusive Convection in a Porous Medium Based Upon the Brinkman-Forchheimer Model, Journal of Mathematical Analysis and Applications, 204 (1996), 138-155.

[19] P. R. Sears, K. Thompon, M. R. Knowles and C. W. Davis, Human Airway Ciliary Dynamics American Journal of Physiology : Lung Cellular and Molecular Physiology, 304(3) (2012), $170-183$.

[20] S. M. Mitran, Metachronal Wave Formation in a Model of Pulmonary Cilia, Computers \& structures, 85 (2007), 763-774.

[21] T. F. Weinstein and L. S. Benethum, On the Derivation of the Transport Equation for Swelling Porous Materials with Finite Deformation, International Journal of Engineering Science, 44 (2006), 1408-1422.

[22] X. Xie, J. Xu and G. Xue, Uniformly-Stable Finite Element Methods for Darcy-StokesBrinkman Models, Journal of Computational Mathematics, 26 (2008), 437-455.

Nattapol Oangwatcharaparkan Department of Mathematics, King Mongkut's Institute of Technology Ladkrabang, Ladkrabang, Bangkok, 10520, Thailand.

E-mail: 63605006@kmitl.ac.th

Kanognudge Wuttanachamsri Department of Mathematics, King Mongkut's Institute of Technology Ladkrabang, Ladkrabang, Bangkok, 10520, Thailand.

E-mail: whychamsri@hotmail.com 